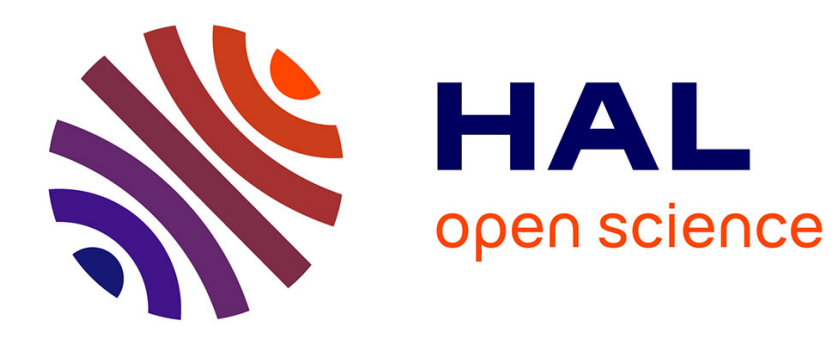

\title{
Dis, dis donc, disons : du verbe au(x) marqueur(s) discursif(s)
}

Juliette Delahaie

\section{To cite this version:}

Juliette Delahaie. Dis, dis donc, disons: du verbe $\mathrm{au}(\mathrm{x})$ marqueur(s) discursif(s). Langue française, 2015, 186 (2), 10.3917/lf.186.0031 . hal-01844679

\section{HAL Id: hal-01844679 \\ https://hal.science/hal-01844679}

Submitted on 19 Jul 2018

HAL is a multi-disciplinary open access archive for the deposit and dissemination of scientific research documents, whether they are published or not. The documents may come from teaching and research institutions in France or abroad, or from public or private research centers.
L'archive ouverte pluridisciplinaire HAL, est destinée au dépôt et à la diffusion de documents scientifiques de niveau recherche, publiés ou non, émanant des établissements d'enseignement et de recherche français ou étrangers, des laboratoires publics ou privés. 


\author{
Dis, dis donc, disons : du verbe au(x) marqueur(s) discursif(s) \\ Juliette Delahaie \\ Université Paris Ouest Nanterre La Défense, Laboratoire Modyco, UMR 7114
}

\begin{abstract}
Résumé
L'objet de cet article est d'analyser les trois formes de l'impératifs de dire, dis/dites/disons, lorsqu'elles sont considérées comme éloignées du sens plein du verbe, dans des énoncés tels que ceux-ci : dis, tu n'as pas vu mes clefs? mais dites donc, il ne faut pas vous gêner! disons que je n'apprécie pas vraiment cette personne. Il y a un problème, disons, un différend entre nous. Il existe un certain nombre d'études qui visent à proposer des taxonomies des contextes d'emploi les plus fréquents de ces formes, classées tantôt parmi les marqueurs discursifs (dis/dites), les marqueurs de glose (disons) ou les interjections (dis donc notamment). A travers une étude des propriétés syntaxiques et sémantiques de ces différents morphèmes, on montrera qu'ils ne peuvent être traités de manière unitaire, car il existe deux entités différentes pour dis/dites et disons, qui fonctionnent soit comme de véritables marqueurs discursifs, soit comme des verbes parenthétiques ou verbes recteurs faibles.
\end{abstract}

Mots clés: sémantique, impératif, dis/dites/disons, verbes parenthétiques, marqueurs discursifs

\title{
English abstract
}

This paper deals with the study of the three imperative forms of dire, namely dis/dites/disons, when they are not used as a verb, as in the following examples : dis, tu n'as pas vu mes clefs? mais dites donc, il ne faut pas vous gêner! disons que je n'apprécie pas vraiment cette personne. Il y a un problème, disons, un différend entre nous. Several studies provide taxonomies of the different uses of these forms which are generally considered as discourse markers (dis/dites), reformulation markers (disons) or interjections (especially dis donc). We will analyse the semantic and syntactic properties of these markers to show that they should not be treated as a unic entity. There are two different realisations of dis/dites and disons, which act as discourse markers, or as parenthetical verbs.

Key words : semantics, imperative, dis/dites/disons, parenthetical verbs, discourse markers

L'objet de cet article est d'étudier quels sont les liens entre les emplois verbaux et les emplois « discursifs » des impératifs de dire, dis/dites/disons, dans des énoncés du type suivant:

(1) a Dis, tu n'as pas vu mes clefs ?/Dites, vous n'avez pas vu mes clefs ?

b. Dis donc, elle est bien ta voiture !/Dites donc, elle est bien votre voiture !

c. Dis-moi, qu'est-ce que tu penses du nouvel informaticien ? / Dites-moi, qu'est-ce que vous pensez du nouvel informaticien?

d. Disons qu'il y a un petit problème/Il y a, disons, un petit problème.

e. Elle est belle, disons plutôt, charmante.

Ces impératifs sont généralement considérés comme distincts du verbe au sens plein, mais la question de savoir à quelle catégorie grammaticale ou fonctionnelle ils appartiennent est loin d'être résolue. Les terminologies sont ainsi assez variées; dis/dites peuvent relever chez certains de la catégorie des interjections (Drescher 2003 à propos de dis donc), des 
«marqueurs discursifs» (Dostie 2004), des «locutions-phrases » (Grevisse et Goosse à propos de dis donc) qui servent à interpeller le locuteur, disons étant généralement étudié en tant que marqueur de glose (Steuckard 2005) ou de reformulation (Saunier 2012). Ce qui frappe avant tout, c'est la multiplicité des contextes dans lesquels ces «marqueurs » apparaissent -nous les nommerons ainsi pour l'instant faute de mieux-, la diversité des formes qu'ils peuvent prendre, par exemple pour dis : dis-moi, dis voir, dis donc et pour disons : disons mieux, disons-le, disons plutôt, et par conséquent la pluralité de leurs valeurs. On peut relier ce phénomène au fait que dire fait partie des verbes nucléaires, c'est-à-dire des verbes de base qui ont un spectre d'emploi relativement large comme aller, écouter, tenir, et qui de ce fait, selon un certain nombre d'études (Dostie 2004 ; Andersen 2007), sont susceptibles de se grammaticaliser/pragmaticaliser pour former des unités plus ou moins figées qui passent dans la catégorie des marqueurs de discours. L'objectif de la plupart des études sur les impératifs de dire est de recenser de la manière la plus exhaustive possible les différentes valeurs du morphème liées à des contextes d'emploi spécifiques. Ainsi, Dostie $(1998 ; 2004)$ propose une description minutieuse de chacun des sens exprimés par dis donc en français québécois, son analyse est orientée vers la description sémantique des marqueurs discursifs en vue de leur analyse lexicographique. Dostie propose ainsi quatre valeurs différentes pour dis donc, qui ont toutes un point commun, c'est de référer à " l'idée d'un fait nouveau que le destinataire est invité à prendre en considération » (1998: 78), même si n'est pas posée $a$ priori l'unicité de sens pour une même forme. Le marqueur disons a lui aussi été abondamment étudié. Steuckardt (2005) le présente comme un «marqueur de glose » qui a des emplois divers en fonction de ses réalisations (disons-le, disons mieux), et Saunier (2012) trouve sept sortes différentes de reformulation pour disons. On utilisera ici ces études mais on proposera une autre approche qui vise plutôt à montrer qu'il existe des distinctions syntaxiques et sémantiques entre les différentes réalisations de l'impératif. Autrement dit, notre hypothèse serait que les différentes valeurs des marqueurs dis/dites/disons, que l'on explique à travers des contextes d'emplois différents, sont en fait les réalisations visibles de d'entités sémantiques différentes, marqueurs discursifs ou autres catégories. On essaiera ainsi de montrer qu'il existe non pas un mais deux dis et disons qui seraient proches par leur fonctionnement, pour l'un des marqueurs discursifs (dis $s_{1}$ et disons $\left.s_{1}\right)$ et pour l'autre des verbes parenthétiques ou verbes recteurs faibles (dis 2 et disons $\left.s_{2}\right)$.

\section{Du verbe au marqueur discursif}

\subsection{Marqueur discursif, marqueur de glose, verbe parenthétique, comment s'y retrouver ?}

Pour tenter d'élucider le sens de ces différentes réalisations de dire à l'impératif, on partira d'une discussion autour de la notion de «marqueur discursif » : quelle définition lui donner, quels sont les critères d'identification ? La dénomination elle-même pourrait être remise en cause, car les verbes qui n'ont plus de capacité rectrice sont souvent désignés par un autre terme que celui de marqueur discursif, comme incise ou verbe recteur faible (BlancheBenveniste 1989, Apothéloz 2003), parenthétique (Andersen 1997) ou marqueur discursif propositionnel (Andersen 2007). On rappellera la définition première du verbe parenthétique née d'une observation de Urmson (1952) et reprise par Blanche-Benveniste (1989) pour définir les «verbes recteurs faibles ». Il s'agit de verbes qui peuvent être utilisés dans deux types de constructions : ils peuvent être en tête de construction, suivis d'une «que-phrase », ou en incise (médiane ou finale), après une séquence $\mathrm{p}$ :

(2) a. Je pense qu'il fait beau demain.

b. Il fait beau, je pense, demain.

c. Il fait beau demain, je pense. 
Dans tous les cas, la proposition $\mathrm{p}$ apporte l'information principale, et le verbe parenthétique a une fonction de modalisation ou d'atténuation (de la valeur de vérité de $p$ ), liée à un affaiblissement $\mathrm{du}$ sens plein ou «fort» du verbe. Nous verrons que les différentes réalisations de l'impératif dis/dites/disons ne répondent pas toutes et exactement à ces descriptions des verbes recteurs faibles ou parenthétiques, l'une orthodoxe (BlancheBenveniste 1989 et Apothéloz 2003 par exemple), l'autre critique (Gachet 2009). Cependant, les critères syntaxiques et sémantiques proposés dans ces études pour définir les verbes parenthétiques, sont tout à fait pertinents pour faire la différence entre ces réalisations des impératifs de dire :

(3) a. Dis que/si tu as volé à la Fnac.

b. Dis-moi/dis-le/dis, tu as volé à la Fnac ?

c. Dis donc, tu as volé à la Fnac !

(4) a. Disons que tu as volé par plaisir.

b. Tu as volé par plaisir, disons par goût du risque.

Si ces constructions peuvent être considérées à première vue comme des constructions parenthétiques, elles partagent aussi un certain nombre de caractéristiques attribuées aux marqueurs de discours. Pour Andersen (2007 : 13-14), ces impératifs sont en fait de véritables «marqueurs discursifs propositionnels » qui partagent les traits suivants avec les marqueurs discursifs mono-syllabiques : invariabilité morphologique, optionalité sur le plan syntaxique, position relativement libre par rapport à un énoncé dans lequel ils sont insérés, aucune contribution au contenu propositionnel de l'énoncé, aucune modification de la valeur de vérité de l'énoncé, sens subjectif ou intersubjectif. Bolly (2010), suivant Brinton (2008), donne même une définition des verbes parenthétiques, qu'elle appelle d'ailleurs «marqueurs parenthétiques ", qui n'a plus rien à voir avec celle de Blanche-Benveniste (1989) et d'Urmson (1952), et où les marqueurs parenthétiques sont totalement apparentés à des marqueurs discursifs. Nous préférons quant à nous garder une définition «traditionnelle » du verbe parenthétique avec tous les défauts qu'elle comporte, et pour cela nous nous référerons à Gachet (2009) qui met fort bien en valeur les points d'achoppement dans les descriptions proposées. En effet, il nous semble qu'il existe une différence certaine entre verbe parenthétique et marqueur discursif, et qui va nous permettre justement de faire la distinction entre plusieurs entités morphologiquement semblables pour dis/dites et disons. Nous verrons ainsi que ces propriétés fort connues des marqueurs discursifs, que l'on retrouve dans la plupart des définitions -larges- de ces derniers (voir par exemple Dostie et Pusch : 2007 ou Somolinos : 2011) ne répondent pas tout à fait aux réalisations de l'impératif de dire qui échappent là encore aux catégorisations existantes ou du moins, en font bouger les lignes.

\subsection{Dis/dites, comment différencier le verbe du marqueur discursif ?}

Nous partons de l'hypothèse qu'il y aurait trois réalisations différentes de l'impératif dis/dites $^{l}$, du verbe dans son sens plein au(x) marqueur(s) discursif(s); on présentera une série de critères qui permettent de faire des distinctions claires entre les différentes formes. La simple application de la propriété syntaxique énoncée plus haut à propos des verbes recteurs faibles donne des indications éclairantes sur leur fonctionnement. Certes, les impératifs

\footnotetext{
${ }^{1}$ On se concentrera par commodité sur l'impératif de la deuxième personne du singulier, tout en signalant, dans les cas nécessaires, les différences qui peuvent exister avec dites à la deuxième personne du pluriel.
} 
dis/dites ne répondent pas aux propriétés d'un verbe parenthétique parce que contrairement à ces derniers, ils peuvent être employés dans une incise antéposée :

(5) a. Dis, tu pars à 9h00 ?

b. Dis donc, elle est belle ta robe !

Cela dit, les critères de positions syntaxique permettent de distinguer entre deux réalisations de dis/dites, selon que le verbe est suivi d'une que $p$ ou d'une si $p$ :

(6) a. Dis-moi si tu pars à 9h00.

b. Tu pars à 9 h00, dis ? dis-moi ?

c. ? Tu pars, dis-moi/dis, à $9 \mathrm{~h} 00$ ?

On peut en effet trouver qu'il y a équivalence sémantique entre (6)a et b, mais (6)c est impossible, sauf lorsqu'il y a reprise du complément par un pronom : Tu les sors, dis-moi, tes enfants le dimanche? Il s'agit d'une première différence avec les verbes recteurs faibles lorsque dire est employé avec la conjonction si d'interrogation indirecte totale ${ }^{2}$. L'écart est encore plus criant lorsque la proposition $\mathrm{p}$ n'est pas une interrogative :

(7) a. Dis donc qu'elle est belle ta robe !

b. Elle est belle ta robe dis donc !

c. Elle est belle dis donc ta robe !

(7)a. n'est pas équivalent à (7)b et $\mathrm{c}$, à moins que l'on considère que en (7)a. comme un morphème exclamatif : dis donc, qu'elle est belle ta robe!

Ce que montrent ces deux séries d'exemples (6) et (7), c'est donc qu'il y aurait deux réalisations différentes de dis. Ce faisant, on s'oppose d'emblée à un traitement unitaire de ces deux formes et à l'idée développée par Andersen (2007 : 22) selon laquelle dis, comme tous les marqueurs discursifs propositionnels de forme impérative, aurait une position préférée : l'antéposition de ces marqueurs discursifs propositionnels serait la position la plus fréquente, car la fonction de l'impératif est d'attirer l'attention de l'interlocuteur sur ce qui suit. Mais ce que montrent surtout nos deux exemples, c'est que l'on a en fait deux comportements distincts de dis. Partant d'une propriété propre aux verbes parenthétiques, et que ne partagent pas nos deux réalisations de dis, on arrive donc d'emblée à une première partition. Il y aurait un $d i s_{1}$ qui serait associé à une demande de dire, équivalent -du moins pour l'instant- à dis si $\boldsymbol{p}$ et non pas dis que $\boldsymbol{p}$. Il s'agirait donc d'un dis introducteur de discours rapporté. Dis 2 en revanche, qui apparaît en contexte exclamatif ou assertif comme en (7), ne correspond pas à dis si $p$, mais n'est pas non plus équivalent à dis que $p$.

Ces deux formes s'éloignent de l'emploi verbal plein dans la mesure où elles ne peuvent être niées. Cette «transparence à la négation » est une caractéristique propre aux marqueurs de discours, mais aussi aux adverbes d'énonciation et aux verbes parenthétiques (voir par exemple Apothéloz 2003 : 245) :

(8) - dis-moi/dis/dis donc, tu pars à 9h00 ?

- non (je ne pars pas à 9h00/mais pas « je ne te le dis pas »)

${ }^{2}$ On notera un fait étrange que nous ne saurions expliquer pour l'instant, c'est que la construction dire si p, mentionnée par exemple chez Riegel et al. (2002: 500), n'est pas répertoriée dans les dictionnaires que nous avons consultés (Littré, TLF et Petit Robert). 
(9) - dis donc elle est belle ta robe

- non, c'est pas vrai (qu'elle est belle)

En (8) et (9), ce qui est nié est «tu pars à 9 h00 », « ta robe est belle », et non dis. En revanche en emploi verbal, on peut nier le fait de dire ou le contenu du dire :

(10) - dis que tu m'as trompé !

- non (je ne te le dis pas/je ne t'ai pas trompé)

Cette insensibilité à la négation permet également d'expliquer l'expression ne me dis pas que en contexte interrogatif. Le fait qu'il s'agisse d'une expression figée est tout d'abord marquée par le fait que la forme positive est impossible :

(11) a. ?Dis qu'il est déjà parti ?

vs b. Ne me dis pas qu'il est déjà parti ?

C'est que ne me dis pas que ne peut pas être compris dans un sens littéral. En effet, l'énonciateur de (11)b suppose qu' "il est déjà parti » (même si ça n'est pas le cas) et que son interlocuteur va confirmer cette supposition. On est dans une sorte de négation rhétorique dans laquelle ne me dis pas que $p$ veut dire je suis sûr(e) que tu vas me dire que p. De plus cette expression, non répertoriée dans les dictionnaires consultés (Littré, TLF, Le petit Robert) est là aussi insensible à la négation :

(12) - ne me dis pas qu'il est déjà parti ?

- non, il n'est pas parti / si, il est parti

- ?? non, je ne te le dis pas / ?? si, je te le dis

A ce stade de notre analyse, nous avons donc distingué deux dis associés à une phrase de type interrogatif ou exclamatif/assertif. La différence entre les deux serait ténue si elle ne s'en tenait qu'à cela. La possibilité de régir ou non un complément, critère que l'on emprunte cette fois aux propriétés des marqueurs discursifs issus d'un verbe, va permettre de rajouter une propriété distinctive entre les deux formes. Une indication forte du degré de figement d'un marqueur discursif propositionnel, c'est qu'il est en effet dépourvu de complément. C'est le cas de la plupart des marqueurs discursifs dont l'origine est verbale, du type regarde/allez, etc (voir Fagard 2012), et dans à peu près toutes les langues romanes. On peut rapprocher cela d'une des propriétés majeures des constructions à verbe recteur faible : la complétive n'y est pas substituable par un pronom, et encore moins avec une construction disloquée. En réalité avec les impératifs de dire, le test est beaucoup moins clair :

(13) a- Dis-moi si tu as volé au supermarché, allez dis-le moi (que tu as volé au supermarché). b. - Dis-moi, tu as volé au supermarché ? Dis-le moi, tu as volé au supermarché, oui ou non?

c. - Dis, tu as volé au supermarché ? Dis-le.

Dis $s_{1}$ peut fonctionner avec un complément en construction disloquée, et il est remplaçable par dis-moi. On pourrait aussi objecter qu'on a là deux réalisations de l'impératif de dire dans un même énoncé, cependant, il y a des cas où cela fonctionne beaucoup moins bien :

(14) a. Dis donc, elle est belle ta robe !

b. ?? Dis-le donc, elle est belle ta robe ! 
(15) a. Dis-moi donc, elle est belle ta robe !

b. ?? Dis-le moi donc, elle est belle ta robe !

On voit bien que (14b) et (15b) sonnent très mal. Par conséquent, seul dis 2 donc répond au critère de l'absence de complément propre aux marqueurs discursifs.

Enfin, on utilisera un dernier critère qui est l'acceptation ou non de modificateur. Les marqueurs discursifs, à la différence du verbe, n'admettent pas de modificateur. Ce dernier critère est repris de Sirdar-Iskandar (1983) qui fait ainsi la différence entre voyons dans son emploi verbal et son emploi interjectif (ibid. : 112). Elle prend l'exemple du modificateur un реu qui ne peut fonctionner que lorsque le verbe est dans son emploi verbal, or là encore, ce critère permet de faire la différence entre deux réalisations de dis :

(16) a. Dis-moi un peu, qu'est-ce que tu as fait hier soir ?

b. Dis un peu, qu'est-ce que tu as fait hier soir?

c. ?Dis donc un peu, qu'est-ce que tu as fait hier soir?

d. ?? Dis donc un peu, tu t'es bien amusé hier soir!

Dis un peu pourrait sembler peu utilisé, mais on en trouve quelques attestations dans Frantext :

(17) Yvonne crie à

Pascal qui est en avant d'elles, accroché à des racines :

«Pascal?

- Quoi donc?

- Dis un peu... quel âge as-tu au juste?

Il grimpe sans les regarder : «Douze ans ! » Il ne ment que de deux mois.

(Aragon, Les voyageurs de l'impériale, 1947)

Il y a en revanche beaucoup d'occurrences dans Frantext de dis-moi un peu :

(18) Quelquefois cependant elle me prenait à part, et me murmurait ce qu'elle n'osait formuler tout haut.

- Dis-moi un peu, quand partirez-vous d'après toi ?

(Duras, Cahiers de la guerre et autres textes, 2006)

Sur la base de ces critères, on peut ainsi faire la distinction entre dis en emploi verbal (dis quelque chose/dis si/que $X$ ) et deux dis en emploi discursif. Le premier dis joue un rôle d'introducteur d'interrogation. Pitavy (2005) parle dans ce cas d' "auxiliaire injonctif d'interrogation », terminologie qui souligne le double statut de dis dans ce type de contexte : tout en gardant sa valeur injonctive, l'impératif y devient « auxiliaire » de l'interrogation qu'il accompagne. Ce dis $s_{1}$, réalisé aussi en dis-moi ou dis donc, a une mobilité limitée dans la phrase, il accompagne uniquement une demande de dire ou de faire (interrogation ou acte illocutoire dérivé), il est difficilement soumis à la négation et il conserve certaines caractéristiques du verbe, notamment le fait d'être modalisable par un peu et de pouvoir être employé avec un pronom. Le second dis 2 , généralement réalisé en dis donc, ne possède plus aucune propriété propre au verbe et possède toutes les caractéristiques d'un marqueur de discours. La différence avec dis $s_{1}$ tient notamment dans le fait qu'il n'accompagne pas forcément une demande. C'est sur la valeur sémantique de ces deux dis que nous nous attarderons maintenant. 


\section{Dis/dis-moi et dis donc, deux marqueurs discursifs différents?}

Au-delà des différences syntaxiques relevées plus haut, qu'est-ce qui différencie dis 1 et dis donc ? Ont-ils la même valeur sémantique ? La question est d'autant plus importante que les marqueurs discursifs à l'impératif sont généralement considérés de manière globale. Ce sont, selon Blum-Kulka (1989), des «attention getters » (capteurs d'attention); selon Andersen (2007 : 24), ils servent à «attirer l'attention de l'interlocuteur sur ce qui suit ». En réalité, la valeur sémantique de ces deux dis est différente, et l'on essaiera d'expliciter leur sens à travers quelques caractéristiques, notamment en étudiant les suites d'énoncés qu'ils peuvent introduire ou accompagner. Tout d'abord, nous l'avions vu plus haut, dis $s_{2}$ donc n'est pas obligatoirement associé à une demande de dire ou de faire, il est même le plus souvent associé à une phrase exclamative :

(19) (Je vois mon amie rentrer chez moi avec une superbe robe ) : «Dis donc elle est belle ta robe !»

On remarquera que dis seul est aussi plus difficile dans ce contexte :

(20) Dis elle est belle ta robe !

Et qu'il appelle plus facilement une demande, ou en tout cas une suite explicite liée à une demande :

(21) Dis elle est belle ta robe, tu l'as achetée où ?

Autrement dit, dis 1 conserve la valeur jussive de l'impératif du verbe au sens plein. On prendra l'exemple de l'énoncé dis-moi si tu as un problème; il s'agit d'une forme d'interrogation indirecte qui correspond à la glose «je te demande de me dire si tu as un problème », dis ayant un effet d'insistance (dis-le moi). Dis $s_{1}$ introducteur d'interrogation conserve ce sens : dis, tu as un problème ? pourrait être équivalent à « je te demande de me dire si tu as un problème ». L'expression dis voir conserve cette valeur :

(22) - Pour rester jeune et vigueuriot comme t'es, comment tu as fait, dis voir ?

- Moi ? Je suis resté filialement collé à la mamelle de la Terre. Je la suce comme l'enfant suce sa mère. » (H. Vincenot, Le Pape des escargots, 1972, Frantext)

Selon le $T L F$ le verbe voir ici en emploi adverbial est placé directement après le verbe à l'impératif qu'il sert à accentuer.

$D i s_{1} / d i s_{1}$-moi est donc un appel à dire ou faire, mais seul dis $s_{1}$-moi implique obligatoirement une verbalisation de ce dire, tandis que dis seul peut être associé à un impératif indiquant une requête, contrairement à dis-moi :

(23) a. Dis, tais-toi maintenant !

b. ?? Dis-moi, tais-toi maintenant !

On serait bien tenté de voir en (23)a. un dis $_{1}$ demande de faire, et pourtant, on ne peut pronominaliser la proposition qui le suit -puisqu'il ne s'agit pas à proprement parler d'une demande de dire-, on ne peut non plus le modaliser par un peu :

(24) a. ? Dis un peu, tais-toi maintenant ! 


\section{b. ? Dis-le, tais-toi maintenant !}

On acceptera beaucoup plus facilement ces transformations avec un acte illocutoire dérivé marqué par le verbe pouvoir par exemple, comme si la verbalisation -sur la capacité- n'était pas totalement oblitérée dans cette demande de faire :

(25) a. Dis-moi un peu, est-ce que tu peux te taire maintenant ?

b. Dis, est-ce que tu peux te taire maintenant, dis-le moi ?

Par conséquent, avec dis $s_{1} /$ dis $_{1}$-moi, on reste obligatoirement dans une demande de dire, et non exclusivement une demande de réagir. $D i s_{2}$ aurait en revanche un spectre d'emploi beaucoup plus large, mais plus qu'une demande de réagir, ce dis 2 possède aussi une autre instruction sémantico-pragmatique. On essaiera d'en expliquer la valeur en partant d'un test utilisé par Somolinos (2003: 74) pour montrer que le marqueur écoute ne peut apparaître en début d'interaction: «il porte sur un énoncé p qu'il marque comme réactif ». Introducteur d'une demande, dis $1 / d i s_{1}-m o i$ peut tout à fait apparaître dans cette position :

(26) (à la secrétaire d'un cabinet médical) «Dites, je suis pressé, je ne peux plus attendre, je décale mon rendez-vous »

En revanche, dis/dites sonne beaucoup moins bien comme réponse à une question, associé à un énoncé réactif :

(27) - comment vous allez faire pour faire baisser le chômage ?

- dites, nous avons mis en place des mesures depuis un certain temps !

- écoutez, nous avons mis en place des mesures depuis un certain temps

Ce n'est pas que dites soit ici impossible mais il n'introduit pas une demande de dire, dites ici sonne comme un reproche, et ce qui pourrait être sous-entendu serait «vous n'êtes pas au courant»? On pourrait d'ailleurs rajouter quelques éléments pour rendre plus clairs les sentiments du locuteur : mais dites donc, nous avons mis en place des mesures depuis un certain temps! Ce n'est plus le dis 1 qui est ici employé, mais le dis $2 / d i s_{2}$ donc. Ce marqueur discursif véhicule en fait l'idée d'un état du monde supposé connu de l'interlocuteur. On essaiera d'en comprendre le mécanisme à l'aide d'un exemple simple :

(28) - tu me donnes un bonbon?

- hé dis/dis donc, tu en as déjà mangé quatre ce matin !

On voit bien comment on passe de dis $s_{1}$ incitatif à dis 2 exclamatif. Dis $s_{1}$ incitatif aurait la glose suivante : « je te demande de dire si $\mathrm{p}$ ». Dis $s_{2}$ exclamatif aurait pour valeur : « je te demande de dire/reconnaître que $\mathrm{p} »$, où $\mathrm{p}$ fait partie des connaissances considérées comme partagées par les locuteurs en présence. Ainsi, on remarquera qu'on pourra difficilement introduire avec dis $s_{2} / d i s_{2}$ donc une information présentée comme entièrement nouvelle :

(29) - tu me donnes un bonbon?

- ? dis/dis donc, désolé, je n'en ai plus.

En revanche, on trouvera plus facilement «écoute, désolé, je n'en ai plus ». Avec $\operatorname{dis}_{2} /$ dis $_{2}$ donc, on est donc dans une réaffirmation de $\mathrm{p}$, d'où son association fréquente avec des 
phrases de type exclamatif. Ainsi, seule la réalisation $d i s_{2} / d i s_{2}$ donc peut-être employée de manière (presque) autonome, en association avec d'autres interjections :

(30) (un passant assiste à un accident dans la rue) : « hé ben dites/dis donc »

Dans cet exemple, impossible de remplacer dis donc par dis ou dis-moi. Pour Dostie (1998 : 77), ce dis donc constitue un acte réactif à une situation ou une assertion apportant une information nouvelle, interprétation contraire à ce que l'on essaie de démontrer. Avec $d i s_{2} / d i s_{2}$ donc marqueur discursif, on est dans la mise en scène de sa propre réaction, glosée par « je te demande de reconnaître que $\mathrm{p}$ », $\mathrm{p}$ étant en quelque sorte considéré comme connu des locuteurs en présence. En effet dans l'exemple précédent, dis donc est bien adressé à un interlocuteur, une personne tierce qui a aussi assisté à l'accident. Le fait qu'il puisse être employé seul indique que $\mathrm{p}$ dans dis donc (p) peut être reconnu, interprété par l'interlocuteur : si je dis dis donc en réaction à un accident, je veux dire quelque chose comme dis donc, quel accident! c'est terrible! et non pas dis donc, quel beau spectacle! Autrement dit, il y aurait une sorte d'énoncé virtuel $\mathrm{p}$ associé à dis donc en vertu duquel cette expression est interprétable par l'interlocuteur, énoncé associé par défaut à une conception stéréotypique ${ }^{3} \mathrm{du}$ monde tel qu'il est conçu à travers la langue.

Enfin, si pour Dostie (1998) la valeur directive de l'impératif se trouve évacuée dans cette dernière réalisation de dis donc, cela ne nous paraît en fait pas si clair : on est plutôt passé de l'appel à dire à l'appel à réagir à mon dire; il s'agit d'une sorte de marqueur de mise en scène du dire qui appelle une réaction quelconque, ce qui explique la fréquence des emplois de dis donc en situation conflictuelle, où le locuteur attend dans notre exemple une réaction (au moins une excuse) de la part de l'interlocuteur :

(31) (un passant me marche ostensiblement sur les pieds) - mais dis donc, il ne faut pas vous gêner!

Ce dernier exemple montre que $d i s_{2} / d i s_{2}$ donc est plus grammaticalisé que $d i s_{1}$ et au détriment de dites $2_{2} /$ dites $_{2}$ donc, dans la mesure où il peut être employé à la place de dites ${ }_{2}$. Ainsi, on pourra avoir :

(32) a. Dis donc elle est belle votre chemise.

b. Dites donc elle est belle votre chemise.

mais pas

c. ? Dites donc elle est belle ta chemise.

Et encore moins

d. ? Dites, tu l'as mise où ta chemise?

En conclusion, on peut distinguer deux formes dis $s_{1}$ et dis $s_{2}$ donc qui n'ont pas les mêmes propriétés syntaxiques et sémantiques, même si dans la plupart des études et des descriptions grammaticales, ces deux formes ne sont pas différenciées. Dis ${ }_{1}$ conserve des caractéristiques du verbe plein et sert à introduire une demande de dire, tandis que dis 2 donc possède toutes les propriétés d'un marqueur de discours qui serait à la fois mise en scène de son propre dire et appel à réagir.

${ }^{3}$ A la suite de Anscombre (1990, 2001), le stéréotype attaché à un terme et qui en constitue la signification est une liste ouverte de phrases génériques communément acceptées par une communauté linguistique donnée. Ces phrases peuvent fonder des enchaînement d'énoncés, elles fonctionnent à la manière d'un thème. 


\subsection{Disons, disons que, verbe parenthétique et/ou marqueur de glose ?}

Plusieurs études ont déjà largement exploré les différentes valeurs de disons, notamment Dostie (2004) pour le français québécois, Steuckardt (2005), Khatchatourian (2008) et Saunier (2012). Pour Grevisse et Goosse (1997 : 468), disons est une espèce de parenthèse par laquelle celui qui parle ou écrit interrompt la phrase par une intervention personnelle. Disons «était au départ une injonction suivie d'un discours direct elliptique : « vous ne pouvez pas venir ce soir? eh bien disons demain ». L'Académie n'admet que cet emploi et déconseille d'utiliser disons en dehors de cette définition précise, qui correspond à " convenons de ». Disons est surtout étudié en tant que marqueur de « reformulation » (Saunier 2012:33) «par lequel on a plutôt une hésitation, avec en jeu une atténuation ». Khatchatourian (2008 : 294) parle de marqueur de dire dans le même sens : «disons est employé dans les situations où le locuteur cherche un mot adéquat (dans le cadre de la dénomination) ou redit ce qu'il a dit auparavant ». C'est selon nous Steuckardt (2005) qui donne la définition la plus précise de ce qu'elle appelle les «marqueurs de glose»: ce sont des marqueurs qui impliquent la "présence de deux énoncés $\mathrm{X}$ ou $\mathrm{Y}$, l'un jouant un rôle de support, l'autre d'apport explicatif ; Y est donné pour une explication de X. » On retrouve une défition semblable pour les marqueurs de reformulation en anglais chez Del Saz et Pennok (2005) à la suite de travaux non édités de Del Saz et Fraser (2003). Seule Dostie (2004) étudie d'autres valeurs de disons, notamment celle de «connecteur textuel» apparenté à une proposition hypothétique en si : "disons que tu as trois enfants, comment tu fais pour finir ton article dans les temps?». Toutes ces études fournissent des descriptions très précises des différents emplois de disons, et là encore, notre objectif sera simplement de montrer qu'il existe au moins deux marqueurs différents qui se rapprochent pour l'un des verbes parenthétiques, et pour l'autre des marqueurs de discours.

Si l'on reprend la propriété fondamentale et définitoire -dans une version classique- des verbes parenthétiques, on pourra d'emblée faire une distinction entre deux réalisations différentes de disons. En (33), disons vérifie toutes les propriétés syntaxiques du verbe parenthétique, et notamment le fait que disons ne puisse figurer en tête de phrase sans que :

(33) - que se passe-t-il ?

- disons qu'il y a un problème entre nous/il y a, disons, un problème entre nous/il y a un problème entre nous disons.

- ?disons, il y a un problème entre nous.

Cependant, il existe des contextes où disons ne peut pas subir de telles transformations syntaxiques sans l'ajout d'autres éléments :

(34) a. La ruse, disons la perfidie, est la meilleure arme politique.

b. ? Disons que la ruse, la perfidie, est la meilleure arme politique.

c. ? La ruse est la meilleure arme politique, disons la perfidie.

Il y aurait donc deux disons dans les exemples (33) et (34). Disons s $_{1}$ serait apparenté aux verbes parenthétiques pourvus d'une valeur mitigeante, le sens de disons étant en effet éloigné du sens « fort » du verbe dire. Il est bien sûr nécessaire de creuser cette valeur d'atténuation et de comprendre en quoi on peut rattacher disons $_{1}$ au sens plein du verbe, mais nous suivrons Gachet (2009) qui montre que l'emploi mitigateur du verbe se superpose à l'emploi descriptif sans l'annuler. Cette conception d'un disons verbe recteur faible ou parenthétique ne rentre 
cependant pas dans la description orthodoxe de ces verbes. En effet, la notion de verbe parenthétique au sens strict renvoie uniquement à des verbes à la première personne du présent de l'indicatif; force est de constater que ces propriétés sont partagées par d'autres formes. En revanche, disons $s_{2}$ de l'exemple (34) fonctionne différemment, et l'on remarquera que l'on peut lui ajouter des modalisateurs ou des compléments :

(35) a. La ruse, disons mieux la perfidie, est la meilleure arme politique.

b. La ruse, disons plutôt la perfidie, est la meilleure arme politique.

c. La ruse, disons le mot la perfidie, est la meilleure arme politique.

Disons $_{2}$ admet donc un certain nombre de modificateurs qui permettent d'exprimer des nuances différentes. Selon Steuckardt (2005: 56), disons le mot «délivre la nomination audacieuse, il met en scène un cheminement menant de la réserve vers un acte de courage linguistique », avec disons mieux, le locuteur y ajoute la «recherche de qualité de la nomination ». Disons $s_{1}$ en revanche, quoique très proche des verbes recteurs faibles au sens classique, admet difficilement de modificateur, ou alors le modificateur fait basculer disons du côté du sens plein du verbe; dans les exemples suivants, disons perd sa fonction de mitigateur pour être glosé par «nous le disons de manière sincère ${ }^{4}$ :

(36) - Disons sincèrement qu'il y a un problème de délai.

- Il y a, disons-le sincèrement, un problème de délai.

Les deux formes se sont éloignées du verbe au sens plein dans la mesure où elles sont toutes deux transparentes à la négation :

(37) - disons qu'il n'est pas très futé

- non, ce n'est pas vrai (qu'il n'est pas très futé),

(38) - la ruse, disons mieux la perfidie, est la meilleure arme politique

- ce n'est pas vrai (que la perfidie est la meilleure arme politique).

Elles n'admettent pas non plus de reprise pronominale de la proposition introduite :

(39) a. ?? Il y a disons, un problème entre nous, disons-le.

b. ?? La ruse, disons mieux la perfidie, est la meilleure arme politique, disons-le.

L'emploi du pronom neutre est un complément figé du marqueur de glose : « cet homme, disons-le, ce voyou, a volé la France ».

Cette courte étude de disons nous a ainsi permis de montrer combien les critères et propriétés distinctives des verbes parenthétiques et des marqueurs discursifs pouvaient être éclairantes pour distinguer, parmi les différentes valeurs et contextes d'emplois de disons, deux réalisations différentes et pourtant souvent semblables en apparence. Disons $s_{1}$ a toutes les caractéristiques syntaxiques et sémantiques des verbes parenthétiques, tandis que disons $s_{2}$ marqueur de glose n'est pas à proprement parler un marqueur de discours, mais plutôt un modalisateur du dire proche d'un fonctionnement adverbial, dont la définition syntaxique et sémantique est encore à faire... La force des deux réalisations tient à l'enrôlement de

\footnotetext{
${ }^{4}$ Gachet (2009) remet cependant en cause la valeur « mitigeante » comme propriété distinctive des verbes recteurs faibles.
} 
l'interlocuteur dans la première personne du pluriel, je/nous + toi/vous, où «l'énonciateur inclut [toujours] le co-énonciateur dans son activité linguistique »(Dostie 2004 : 164).

\section{Comment relier ces différentes réalisations ?}

\subsection{Un processus de grammaticalisation?}

Le lien entre les différentes réalisations de ces impératifs peut s'expliquer en termes de grammaticalisation. Ainsi pour Dostie (1998), l'impératif dis a subi un processus de grammaticalisation dont l'aboutissement est le marqueur discursif dis $s_{2}$ donc, marqué par la décatégorisation grammaticale, un figement et une perte de flexibilité syntaxique. Selon Bolly (2010) qui étudie le processus de pragmaticalisation ${ }^{5}$ du marqueur discursif tu vois, un tel processus est favorisé d'une part, par le sémantisme large du verbe, et d'autre part, par l'augmentation de certains contextes d'emploi, à savoir les emplois en incise. Ainsi, Bolly (2010) montre que tu vois est devenu marqueur discursif en corrélation avec une augmentation de la fréquence des structures en incise (comme tu vois) à la période prémoderne, si bien que «c'est le contexte formel d'apparition qui favoriserait l'apparition de nouveaux sens » (ibid. : 689). On peut dessiner un tel processus pour nos impératifs de dire :

- Dans la première étape, les impératifs de dire se construisent avec que $P$ ou si $P$ et conservent le sens plein du verbe.

- Dans une deuxième étape, ils seraient employés sans complément propositionnel, dans des structures parenthétiques, l'incise étant un phénomène reconnu dans la processus de grammaticalisation. Par élargissement des contextes d'emploi, ces formes subissent un glissement sémantique (atténuation notamment pour disons $s_{1}$ et ${ }_{2}$, sens néanmoins proche de celui du verbe plein pour $\left.d i s_{1}\right)$. A ce stade, les impératifs sont employés dans le domaine textuel : dis 1 sert à introduire une interrogation, disons $s_{1}$ (que p) introduit la proposition $\mathrm{p}$.

- Dans une troisième étape, les formes à l'impératif, dont le sens s'est éloigné de celui du verbe plein, n'appartiennent plus du tout à la structure phrastique, comme dis $d o n c$.

Ce processus diachronique semble en apparence pouvoir fonctionner, même si ce n'est pas très clair pour disons étant donné que cette forme n'a pas produit, en tout cas pour le moment, de marqueur discursif à proprement parler. En réalité, cela n'explique pas tout pour dis non plus. En effet, on a vu qu'il était difficile de rattacher dis 2 donc à l'impératif de dire et à dis dans le sens où dis $s_{2}$ donc ne renvoie pas à un «dis si $\mathrm{p}$ », mais à un «dis que $\mathrm{p}$ ». Les deux réalisations se rattacheraient donc à deux sens différents de dire, celui de la demande et celui du dire. De plus, ce système à trois formes existe dès l'ancien français. Ainsi, l'emploi sans complément de dis est d'un usage très ancien en français :

(39) 1744 À moi apertient franchement.

1745 Or dites donc, si vous gart Dieu,

1746 Se je faisoie .I. nouviau gieu :

(Digueville Guillaume De, Le Pèlerinage de vie humaine, 1330-1331, Frantext)

Ce dis est répertorié dans le Dictionnaire de Jean Nicot (1606) : « Di moy, Narra mihi ». Dans cet emploi étudié par Oppermann-Marsaux (2012), l'impératif $d i$ est suivi d'une proposition interrogative :

\footnotetext{
${ }^{5}$ On définira la « pragmaticalisation » comme le processus de grammaticalisation qui voit des unités lexicales ou grammaticales migrer, au cours des siècles, vers la sphère pragmatique du discours, sans qu'il y ait obligatoirement changement de catégorie grammaticale.
} 
(40) «Di, feras-tu autre cose? » « Dis, feras-tu autre chose ?» (Gautier d'Arras, Eracle, v. 5991)

(41) «Renart, faist il, biax douz conpaig, / di moi, ies tu or ça dedanz? » «enart, dit-il, mon cher compère, dis-moi, es-tu à présent là à l'intérieur ? »(Le Roman de Renart) ${ }^{6}$

Ce dis introducteur d'interrogation, demande de dire, est répertorié tel quel dans les dictionnaires comme le Littré :

Dire s'emploie quelquefois à l'impératif pour appeler l'attention.

"Dites-moi, venez-vous dîner avec moi? D'où vient donc, je vous prie, un tel emportement! Avez-vous, dites-moi, perdu le jugement ?" [Molière, Mis. IV, 3]

"Dis, penses-tu qu'un jour mon père nous pardonne?" [Ducis, Othello, I, 8]

Ce dis au sens 1 est de plus assez répandu dans les langues romanes, on le trouve en italien dimmi, et de manière plus sporadique en espagnol, dime -qui lui préfère oye. Plutôt que d'y voir le résultat diachronique d'un processus de grammaticalisation, on pourrait ainsi considérer dis ${ }_{1}$ et disons ${ }_{1}$ comme des emplois particuliers du verbe, dont ils ont gardé des caractéristiques. Seul $d i_{2}$ donc serait l'aboutissement d'un processus de pragmaticalisation : d'une demande de dire, on passe à une demande de réagir ; d'un appel à autrui, on passe à une mise en scène de son propre dire. Ce dis $s_{2}$ donc est en effet un marqueur assez récent; jusqu'au français classique, il n'existe pas, mais en réalité il possède un équivalent en ancien français, di va, étudié par Oppermann-Marsaux (2014). On remarquera que le lien avec le verbe au sens plein est relativement ténu, dans le sens où de plus, dis $s_{2}$ donc n'a pas d'équivalent littéral dans les langues (voir Bertrand : 2006). Force est de constater que ces formes résistent à l'analyse, et que dis donc seul, en français contemporain, pourrait faire l'objet d'une longue étude...

Bibliographie

ANDERSEN, H.L. (1997), Les propositions parenthétiques en français parlé, Thèse de Ph.D. : Université de Copenhague, non publié.

ANDERSEN, H.L. (2007), « Marqueurs discursifs propositionnels », Langue française, 154, 1328.

ANSCOMBRE, J-C. (1990), « Thème, espace discursif et représentation événementielle », in J.C. Anscombre et G. Zaccaria (éds.), Fonctionnalisme et pragmatique. A propos de la notion de thème, Milan : Unicopli, 43-150.

AnscombRe, J.-C. (2001), « Le rôle du lexique dans la théorie des stéréotypes », Langages, 142, 57-75.

APOTHELOZ, D. (2003), «La rection dite «faible»: grammaticalisation ou différentiel de grammaticité ?», Verbum XXV/3, 241-262.

Bolly, C. (2010), «Pragmaticalisation du marqueur discursif tu vois. De la perception à l'évidence et de l'évidence au discours », in F. Neveu, V. Muni Toke, J. Durand etal. (éds.), Congrès mondial de linguistique française, 673-693. (http://www.linguistiquefrancaise.org) BLANCHE-BENVENISTE, C. (1989), «Constructions verbales < en incise > et rection faible des verbes », Recherches sur le français parlé, 9, 53-74.

BERTRAND, Y. (2006), «Traduire dis donc», Nouveaux cahiers d'allemand, vol.24, $\mathrm{n}^{\circ} 2$, p.175-190.

Blum-KulKa, S., House, J. et KASPeR, G. (éds.) (1989), Cross-cultural Pragmatics :

${ }^{6}$ Cité par Oppermann-Marsaux (2012) 
Requests and Apologies, Norwood, N.J. : Ablex Publishing Corporation.

Brinton, L. J. (2008), The Comment Clause in English. Syntactic Origins and Pragmatic Development, Cambridge : Cambridge University Press.

Del Saz Rubio, M.M. et PenNock SPECK, B. (2005), « Discourse markers of reformulation from the perspective of grammaticalization », Prespectivas interdisiplinares de la lingüistica aplicada, 2, 89-100.

Del SAZ Rubio, M.M et Fraser, B. (2003), Reformulation in English, non publié.

DiCTIONNAIRE Le grand Robert de la langue française (1985), Paris, Le Robert.

DiCTIONNAIRE Trésor de la langue française (1983), Paris.

DostiE, G. (1998), «Deux marqueurs discursifs issus de verbes de perception: de écouter/regarder à écoute/regarde », Cahiers de lexicologie, 73 : 2, 125-146.

DostiE, G. (2004), Pragmaticalisation et marqueurs discursifs, Bruxelles : De BoeckDuculot.

Dostie, G. et Pusch, C. (2007), «Les marqueurs discursifs. Sens et variation. Présentation », Langue française, 154/2,

DRESHER, M. (2003), «ah dis donc les vieux souvenirs. French interjections and their use in discourse ", in Niemeier, S. et Dirven, R. (éds.), The language of emotions, Amsterdam : Benjamins, 233-246.

FAGARD, B. (2012), «E vidha, olha...: Imperatives as discourse markers and grammaticalization paths in Romance : a diachronic corpus study », in P. Lauwers, G. Vanderbauwhede et S. Verleyen, Pragmatic markers and pragmaticalisation : lessons from false friends, Amsterdam : J. Benjamins, 117-140.

GACHET, F. (2009), «Les verbes parenthétiques : un statut syntaxique atypique ? », Linx, 61, 13-29.

GREVISSE Maurice (1997, 13e éd. revue par André Goosse) : Le bon usage, Louvain-la-

Neuve, Duculot.

KHATCHATOURIAN, E. (2008), «Les marqueurs de reformulation formés à partir du verbe DIRE», in M.C. Le Bot, M. Schuwer et E. Richard (éds.), La reformulation. Marqueurs linguistiques et stratégies énonciatives, Rennes : Presses Universitaires de Rennes, 19-33.

OPPERMANN-MARSAUX, E. (2012), «Di de l'ancien français au français classique », in C. Guillot, B. Combettes et al., Le changement en français, études de linguistique diachronique, Berne : Peter Lang, 265-280.

OPPERMANN-MARSAUX, E. (2014), «Les emplois du marqueur discursif dea du moyen français jusqu'au français classique », in J.C. Anscombre, E. Oppermann-Marsaux et A. Rodriguez Somolinos (éds.), Médiativité, polyphonie et modalité en français : études synchroniques et diachroniques, Paris : Presses Universitaires de la Sorbonne, 179-196

PITAVY, J.C. (2005), « dire entre verbe opérateur et discours en grec, latin et français », Revue de sémantique et pragmatique, 17, 137-150.

Riegel, M, Rioul, R. et Pellat, J.C. (1994/2002), Grammaire méthodique du français, Paris : PUF.

SAUNIER, E. (2012), « Disons, un impératif de dire ?», L'information grammaticale, 131, 25-34.

SIRDAR-ISKANDAR, C. (1983), «Voyons ! », Cahiers de linguistique française, 5, 111-130.

SOMOLINOS RODRIGUEZ, A. (2003), «Un marqueur discursif du français parlé : écoute ou l'appel à la raison », Thélème, Revista Complutense de Estudios Franceses 71, Numero extraordinario, 71-83.

SOMOLINOS RodRigueZ, A. (2011), «Présentation : Les marqueurs du discours - approches contrastives », Langages, 184, 3-12. 
SteucKardT, A. (2005), «Les marqueurs formés sur dire », in A. Steuckard et A. NiklasSalminen (éds.), Les marqueurs de glose, Aix en Provence: Publications de l'université de Provence, 51-65.

URMSON, J.-O. (1981), You know: a discourse-functional approach, Amsterdam : Benjamins. 